\title{
Eye For An Eye
}

\author{
David Lyons \\ School of Arts Media and Computer Games \\ Abertay University \\ Dundee, Scotland, DD1 1HG \\ d.lyons@abertay.ac.uk
}

David R. Flatla

School of Computing

University of Dundee

Dundee, Scotland, DD1 4HN

d.flatla@dundee.ac.uk

\begin{abstract}
Artist David Lyons and computer scientist David Flatla work collaboratively to create art that intentionally targets audiences of varying visual abilities mediated through smart device interfaces. Conceived as an investigation into theories and practices of visual perception, they explore the idea that artwork can be intentionally created to be experienced differently dependent on one's visual abilities. They have created motion graphics and supporting recolouring and colour vision deficiency (CVD) simulation software. Some of the motion graphics communicate details specifically to those with colour blindness/CVD by containing moving imagery only seen by those with CVD. Others will contain moving images that those with typical colour vision can experience but appear to be unchanging to people with CVD. All the artwork is revealed for both audiences through the use of specially programmed smart devices, fitted with augmented reality recolouring and CVD simulation software. The visual elements come from various sources, including the Ishihara Colour Blind Test, movie marques, and game shows. The software created reflects the perceptual capabilities of most individuals with reduced colour vision. The development of the simulation software and the motion graphic series are examined and discussed from both computer science and artistic positions.
\end{abstract}

Colour perception. Motion graphics. Colour vision deficiency (CVD). Colour blindness. Mobile devices.

\section{INTRODUCTION}

Eye For An Eye is a continuing exploration of visual stimuli that aims to simultaneously engage audiences of varied colour vision acuities. Artist David Lyons and computer scientist David Flatla worked collaboratively to create art that intentionally targets audiences of varying visual abilities mediated through smart device interfaces. David Lyons is an artist interested in tools that might better help him create art that engages audiences of various sighted abilities. David Flatla is a computer scientist working with colour vision deficiency (CVD, also known as dichromacy or colloquially colour blindness) simulators and is interested in the artistic application of his work.

Conceived as an investigation into theories and practices of visual perception, Eye For An Eye explores the idea that artwork can be intentionally created to be experienced differently, dependent on the viewer's visual abilities. The concept evolved from David Lyons' series of prints: The Double Blind Test Series (Lyons 2013). Visually and tactilely expressive, this series of 12 prints conveyed artistic intentions to the sighted, the blind, the colour blind, and the partially sighted. In Eye For An Eye, these ideas have been developed in collaboration with David Flatla to create a series of motion graphics for audiences with typical colour vision as well as audiences with CVD. Digital simulations, in real time on smart devices, will be used to share those differing experiences with audiences not typically able to view them.

This paper outlines the scientific work and initial experiments in the creation of these motion graphics and the simulation software for those with full colour vision and those with CVD.

\subsection{Art and CVD}

While art created specifically for those with CVD is uncommon, art created by those with CVD is not, and some has been very well received. Expressionist painter and printmaker Clifton Pugh had protanopia (Cole \& Harris 2009). He was made Officer of the Order of Australia for service to Australian Art in 1985 (Australian Government 2014). As speculated by Japanese medical scientist Kazunori Asada, Vincent Van Gogh's vibrant use of colour was due to deuteranopia rather than Fauve influences (Asada 2011). In contrast, in The Case of The Colorblind Painter, Oliver Sacks chronicles an artist who gives up painting after losing his colour vision in a car accident; because of the lack of colour, the artist found his art was without meaning (Sacks 1987). 
The Double Blind Test Series of prints was based on the imagery, layout and colours of the Ishihara tests for colour blindness (Ishihara 1917). Anonymous Play (Figure 1) from the series, strove to make the number six visible only to those with red-green CVD. When exhibited, the print was positively received and drew those with CVD, including Flatla, to the exhibition. A desire to refine this technique for creating differentially viewable artwork and to develop a body of art that can engage multiple audiences simultaneously led to Lyons collaborating with Flatla to develop CVD software simulation that specifically addressed his artistic requirements.

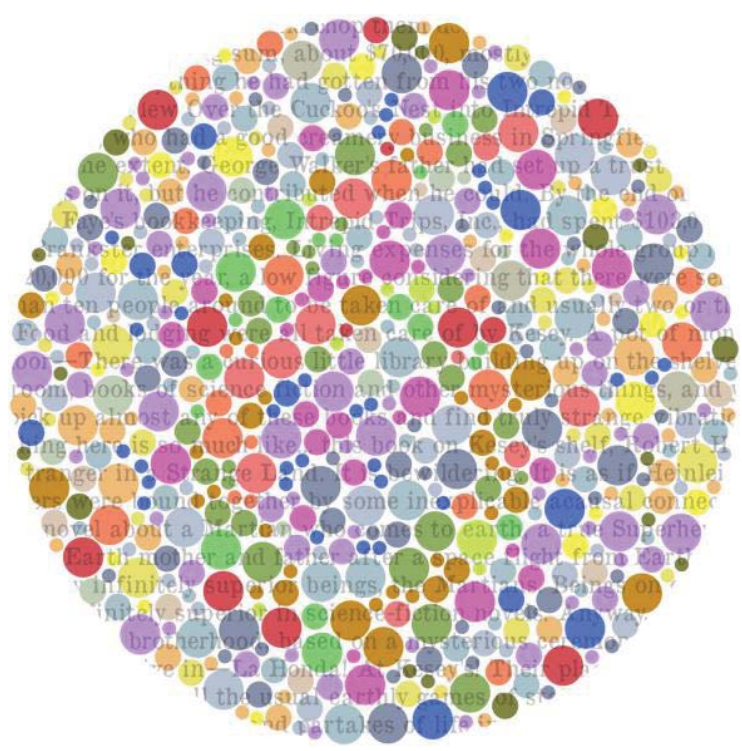

Figure 1: Anonymous Play, from The Double Blind Test Series, 2013

\section{THE CONCEPT}

The pieces in Eye For An Eye also reference the circles of the Ishihara test, as well as the movie marque representations in the intro of The Rocky and Bullwinkle Show (The Bullwinkle Show 2011) and the stage set of the Gong Show (The Gong Show 2006). In movie marques, lights are turned on and off in a sequence to create the illusion of motion. The ambition with this work was to sequentially change the colours of the Ishihara circles to create the illusion of motion, and to manipulate the colours to create motion viewable differently for fully sighted and CVD audiences. The shared culture, accessibility and technology of midto-late twentieth century television and movies complement the accessibility and sharing of visual perception offered by the simulation software on smart devices. The use of the Ishihara imagery reminds the viewer that their visual experience of the work may be different than that of others. Unlike the movie marquee lights, the circles in the pieces do not turn on and off, but change colour instead. The viewer is neither included nor excluded depending on their colour vision abilities, but has a different experience because of it. The software allows those experiences to be shared.

Simulating how an image appears to someone with CVD allows people with typical colour vision to experience the image as though they had CVD. CVD causes a reduction in the number of colours perceived by an individual, such that colours that are distinct to people with typical colour vision are no longer distinct for people with CVD. For example, pink, grey, and turquoise are distinct colours for most people, but for those with protanopia (missing long-wavelength cones), certain varieties of these colours are not differentiable. To simulate the CVD-appearance of an image, the colours in the image must be replaced with colours that are perceived by someone with CVD. Techniques for determining how colours are replaced have been previously described (Meyers \& Greenberg 1988, Brettel, Viénot \& Mollon 1997, Flatla \& Gutwin 2012), so we will describe the general approach briefly in terms of a simplified colour representation.

Colours can be conceptualised using a spatial representation in which individual colours occupy a unique location within the space. For the purposes of describing CVD simulation, consider an additive 3D colour space defined by three orthogonal dimensions: a red-green axis, a blue-yellow axis, and a black-white axis (see Figure 2). A colour's constituent amounts of redness or greenness, blueness or yellowness, and whiteness or blackness specify where the colour is located within this colour space. For example, red will be located at the red extreme of the red-green axis and in the middle of the blue-yellow and black-white axes.

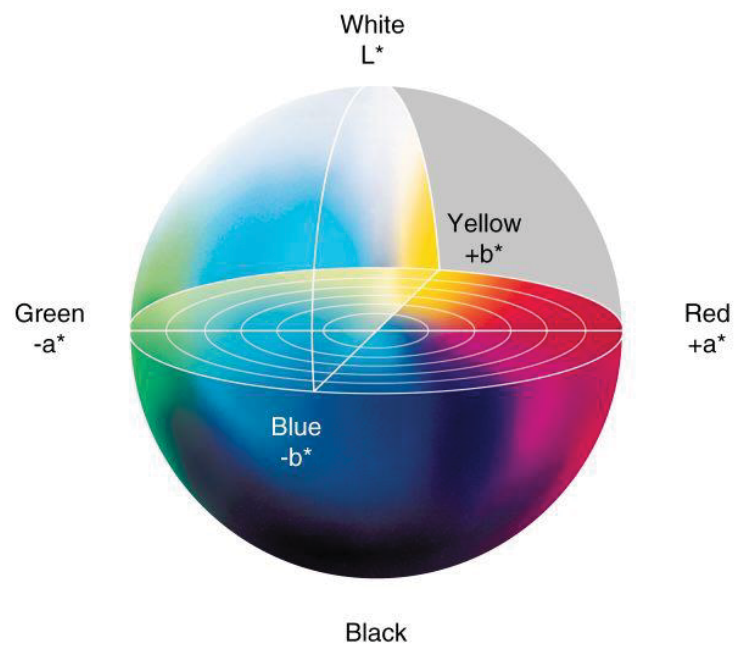

Figure 2: A spatial representation of colour using the CIE $L^{*} a^{*} b^{*}$ colour space where the red-green axis is the $a^{*}$ axis, the blue-yellow axis is the $b^{\star}$ axis, and the blackwhite axis is the $L^{*}$ axis 
Deuteranopia (missing medium-wavelength cones) and protanopia (missing long-wavelength cones) are colloquially called red-green colour blindness; people with these types of CVD have reduced ability to distinguish between greens and reds. In the colour space just described, these CVDs can be represented by collapsing the space along the red-green axis, such that all colours that lie on any line that is parallel to the red-green axis are represented by a single colour that lies on the plane defined by the blue-yellow and black-white axes. In the example given above, the pink, grey, and turquoise that are not differentiable for people with protanopia all lie along one of these lines, so are perceived as the same shade of grey.

\section{THE TOOLS}

\subsection{Motion graphics creation tools}

Most of the stills for the imagery were initially created and tested in Adobe Illustrator. The colour tools in Illustrator were used to identify the RGB percentages for each colour used. The Color Blind Proof setting in the View menu was set to deuteranopia providing a simulation of CVD (it can also be set to simulate protanopia but this was not used here). The Illustrator Deuteranopia Proof setting worked well in giving an initial overview of how the imagery appears to those with this condition, but did not provide the RGB (red, green, blue) percentages of the simulated colours needed to recreate them.

The Mac OS X utility DigitalColor Meter was used to provide colour readings in RGB percentages for the screen area under the cursor, while designing in Illustrator's Color Blindness Proof setting. This was necessary because the colour information displayed in the Illustrator Color Palette is for the original colour, not the simulated deuteranopic colour. The images created in Illustrator were imported into Adobe Premiere to create the motion.

As most existing simulators only work with static images, a prototype desktop implementation of Flatla's CVD simulation software (described in Section 3.2) was used to view a CVD simulation of the motion images. The application rendered visible motion graphics simulated as deuteranopia. Quick key commands allowed easy switching between simulation types.

\subsection{CVD simulation software}

To simulate the appearance of images for people with CVD, CVD simulation software performs the colour space compression described above. People with deuteranopia and protanopia (colloquially called red-green colour blindness) have reduced ability to distinguish between greens and reds. The CVD simulation is achieved by shifting the original colour of each pixel along the line parallel to the red-green axis identified above until it meets the plane defined by the blue-yellow and black-white axes. This new colour is then used to replace the original pixel colour to generate the simulated image.

\subsection{Recolouring for CVD}

Recolouring modifies the contents of an image to improve colour differentiability for people with CVD. This is accomplished by replacing the colours that present difficulties for people with CVD with more differentiable alternatives. For example, suppose an image contains red raspberries against green foliage (a colour combination that will present difficulties for someone with CVD). A recolouring tool maps the colour of the berries to another colour that is more differentiable for someone with CVD (e.g., blue). This introduction of a false colouring scheme restores the perception of colour differences for people with CVD, typically at the expense of preserving the naturalness of the image - blue raspberries are clearly not the norm.

Previous work used dynamic (Jefferson \& Harvey 2006) and static (Seewald Solutions 2013) approaches for choosing replacement colours during recolouring. Dynamic recolouring involves analysing the image to identify its main constituent colours, then finding a set of replacement colours that maximally satisfies a set of constraints typically through constraint optimisation. This approach can help reduce the degree of recolouring that takes place, but is quite computationally demanding. Static approaches rely on a fixed recolouring map, where each possible input colour maps to a pre-determined replacement colour. Static recolouring can tend to result in too much recolouring (e.g., every colour changing), but can be executed very quickly using parallel shaders on the graphics card.

A static recolouring technique was used primarily because of its reduced computational requirement. This allows the recolouring tool to be deployed on a mobile device that allows participants to fully engage with the exhibit.

To construct the static recolouring technique, a mapping of input colours to output colours was developed. To accomplish this, our recolouring tool rotates the spatial colour representation described above by mapping reds to greens, greens to blues, and blues to reds. As discussed above, this results in a large degree of recolouring (see Figure 3), but also allows colours that are not differentiable in redgreen CVD to become differentiable. For the purposes of this work the point of the recolouring tool is to allow the colours that are not differentiable 
for people with CVD to become differentiable so they can appreciate what the participants with typical colour vision perceive. Performing such a severe recolouring guarantees that colour differentiation will be possible for people with CVD.

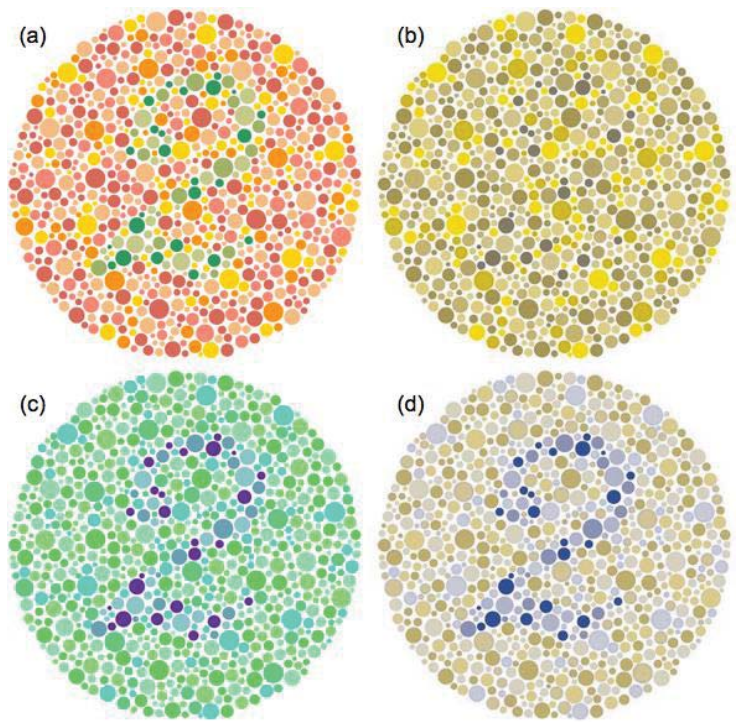

Figure 3: Recolouring demonstration: (a) original Ishihara plate, (b) deuteranopic simulation of the original plate, (c) original plate after recolouring, (d) deuteranopic simulation of recoloured plate

\subsection{Generating look-up-tables for simulations}

Both the simulation and recolouring techniques presented above were described in terms of single images, but to create a mobile application that provides simulated and recoloured views of the world at interactive frame rates, these techniques need to be extended to run in near real time. To do this, the fundamental algorithm used in both simulation and recolouring has been explored to identify where performance can be improved.

At a high level, both simulation and recolouring use almost identical algorithms:

(i) Identify a pixel's RGB colour.

(ii) Manipulate the RGB colour to find its simulated or recoloured version.

(iii) Apply the manipulated RGB colour to the original pixel.

The first and third steps are executed very quickly, so the second step is the bottleneck in the algorithm; both simulation and recolouring involve substantial manipulation of the input RGB colour. These include converting from RGB into other colour spaces that better support the compression performed during simulation and the rotations performed during recolouring, performing the actual colour compression and rotations, and converting the resulting colours back to RGB colour space so they can be applied to the pixel in step three.
To improve the speed of the above algorithm, step two is replaced with a single memory lookup. The simulation and recolouring techniques described above are both deterministic - the same RGB input colour will always result in the same output RGB colour. As the set of RGB colours is fixed (typically with $2^{24}$ unique RGB colours), then the simulation and recolouring colours for every possible input RGB colour can be computed and stored in a LookUp-Table (LUT). The input RGB colour is used as an index into the appropriate LUT (simulation or recolouring), from which the replacement RGB colour is read and used in step three.

To generate the LUTs used for the mobile application used in the exhibit, a single image containing every possible RGB colour was generated, and organised such that a colour's location in the image is dictated by the colour's RGB values. For example, the brightest blue in RGB is represented by 0x0000FF (hex) $=255$ (decimal). The location of this blue in the LUT is at index 255. This image was passed through an implementation of the CVD simulation tools described earlier (Flatla 2012) to get the CVD simulated view of this image. Once through the simulation, the image colour at a particular RGB input location is no longer the input RGB colour, but the simulation colour. As a result, a single memory lookup can be used to go from input RGB colour to output RGB colour. Two simulation LUTs were generated - one for each type of red-green CVD (protanopia and deuteranopia).

To build the LUT for the recolouring tool, an image similar to the CVD simulation LUT image was computationally generated. For each possible RGB colour, the recolouring rotation was performed as described above. The resulting rotated colour was then stored in an output image at the original colour's index. In this fashion, a LUT of exactly the same organisation as the simulation LUTs was generated, allowing the mobile implementation to take advantage of these similarities.

\subsection{Smart device development}

To develop the mobile application, the LUTs generated in the previous section were loaded into a folder on the mobile device. We developed an app that looks in this folder when launched and loads all of the LUTs present into memory. On launch, the filename of each LUT is also read, and stored for later use. When the app finishes loading the LUTs, a video feed from the rear-facing camera is started and fed through to the device's screen. The initial screen presents the camera feed without manipulation and a text label indicating no manipulation is being performed (Clear) is displayed in the corner of the screen. To use a simulation or the recolouring, the user swipes their 
finger across the screen. This switches to the first CVD simulation. At the same time, the text label on the screen is updated to match the filename of the current LUT in use (one of Protanopia, Deuteranopia, or Recolouring). The LUTs are presented in a circular fashion so if the user swipes past Recolouring, they are presented with the Clear screen again. For the exhibition, this app was implemented using the Android ADK, and deployed on a Google Nexus 7 Tablet.

\subsection{Test Panel}

Two local participants viewed images during development, one self-identified with red-green CVD and the other with typical colour vision. Once obtaining the desired results from the local participants, prototypes were emailed to ten additional participants. They were asked to identify any letters, numbers or patterns seen in the motion graphics. They were then asked to name the colours in the graphic. Responses were collated and adjustments were made in response. Once completed, the process was repeated until appropriate results were achieved. When finished, we tested the motion graphics with four participants (two CVD and two not CVD) all with positive results. It is hoped that additional responses can be recorded during the public exhibition and viewing with the simulation enabled smart devices.

\section{THE ART}

\subsection{Testing the colouring technique}

The CVD simulation motion graphic concept was first tested using a page of thirty-three evenly spaced dots of seemingly random colour placement (see Figure 4). To get a variety of hues, blue, red, yellow, green and orange were selected from the Adobe Illustrator web colours with magenta and light blue added to provide variety. The Illustrator proof tool was used to determine what colours the red and green changed to in the deuteranopia mode. A single light-brown hue, between what was represented for the red and green, was selected to replace the red and green dots in the image.

In the image, the differently coloured dots were at first arranged randomly. The deuteranopia mode was then turned on and the dots re-arranged such that those that were yellow and light brown in hue formed a pattern matching the number six. When the deuteranopia mode was switched back off the dots still looked randomly placed. The local CVD test participant was asked if he could see a number within the dot layout and correctly identified it as six. The local participant with typical colour vision was unable to see the six.

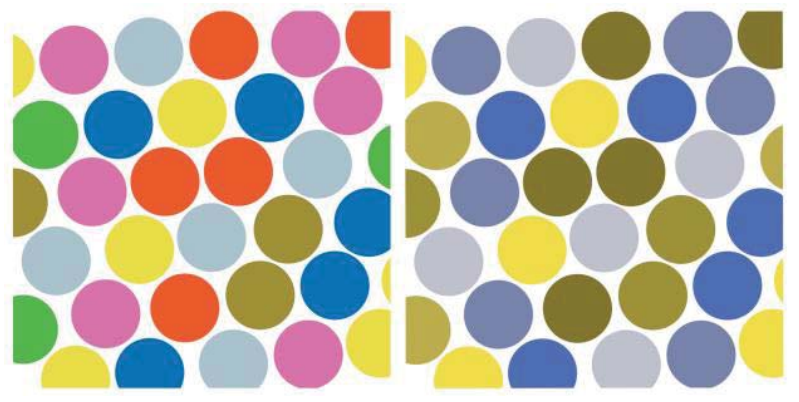

Figure 4: Early attempt at camouflaging visual information. Those with CVD might make out a ' 6 ' in yellows and browns. Original colour version on the left. CVD simulation on the right

\subsection{Testing the sequencing technique}

The next test was inspired by the circles and sequencing lights seen in movie marques and vintage Las Vegas signage. Simple square grids of up to 100 circles against a black background were created in Illustrator. An on/off sequence of three with the circles radiating out from the centre: off, on, on, off, on, on, etc. was created, with off corresponding to black and on to white. A still of this sequence is shown in Figure 5.

Three image iterations of this were created: (i) with the sequencing starting in the centre, (ii) with it starting one row out from the centre and (iii) with it starting two rows out from the centre. These three iterations covered all possible permutations of colour within the off-on-on sequence.

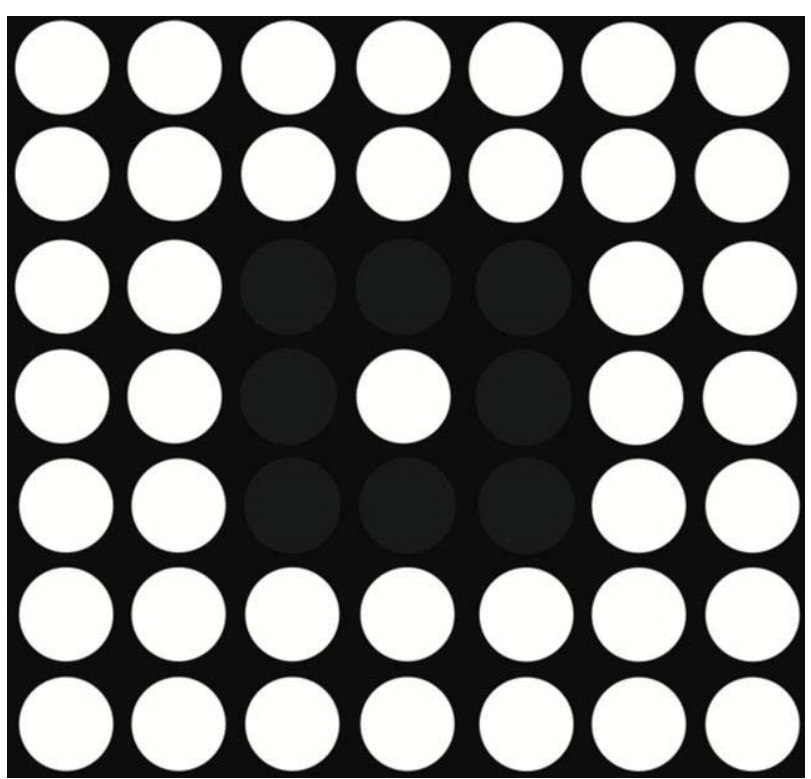

Figure 5: Still of on-on-off sequence from initial motion graphic tests

The three iterations were saved and placed in their correct order into Adobe Premiere video editing software. Each iteration was given six consecutive frames. The three images times six frames were 
then copied and pasted multiple times into the project window of Premiere. When played, the sequence gave the impression of a square rapidly radiating out from the centre of the image.

\subsection{Creating the art}

The third series of tests also mimicked Ishihara imagery as their starting point. Because the circles in the Ishihara imagery vary in size and placement, concentric circles were overlaid on the imagery. If the centre of an Ishihara circle fell within a specified concentric demarcation, then that circle was included within that demarcation. This allowed for less rigid outcomes that better reflected the Ishihara characteristics. Aesthetic expression was more thoroughly explored in this test and required much greater precision in colour selection; which colours those with typical colour vision would see and their relationship to the colours perceived by those with CVD.

The intent with the initial motion test was to have a green number 27 against a red-orange background with a circular pattern radiating from the image's centre. Both the number and the pattern would be visible to people with typical colour vision, but only the radiating pattern would be visible to those with CVD. The colours had to be carefully chosen so that the various reds would correspond with various greens exactly, producing the same exact brown when viewed by those with CVD.

In Illustrator, green and red hues of similar saturation and value were selected and used to fill in two circles. Switching to the Color Blind Proof setting in Illustrator rendered the colours in the brown tones of CVD. From these two, the green simulated brown hue was chosen and the DigitalColor Meter was used to determine its RGB percentage reading. A third circle was filled brown using the RGB percentages in the Color Picker. To generate the same brown hue from the red circle, the RGB percentages of the original red hue were manually adjusted until the correct brown hue was visualised through the CVD simulator. This entailed slightly changing one of the RGB percentages, switching to the Color Blind Proof setting and getting a reading in the DigitalColor Meter to see if it adjusted closer or further to the percentages needed for the red hue to match the desired brown hue. This was repeated until the red and brown hues matched (see Figure 6). The entire procedure was used to produce two matching sets of red, green and brown hues. This manual process was streamlined by the production of an RGB percentage table of reds and greens that collapse to the same browns. This table was used to generate the hues for subsequent pieces of work.

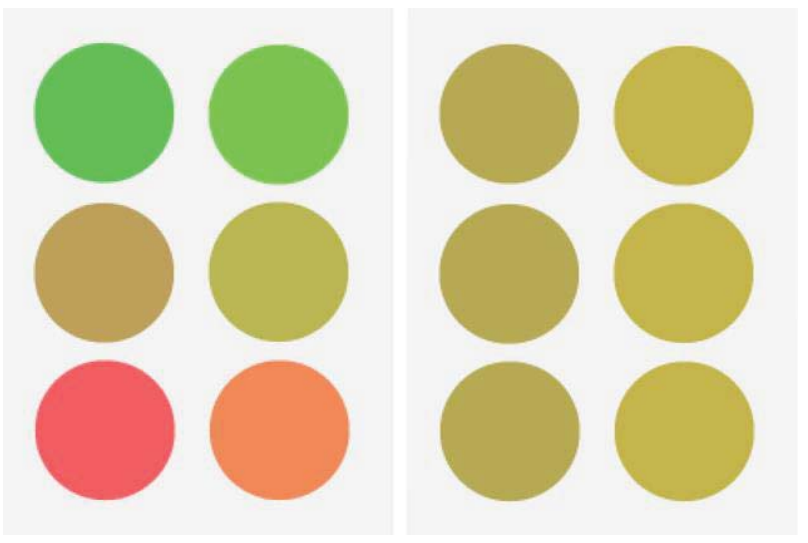

Figure 6: Colour for early Ishihara imagery motion graphic test. Original colours on the left. CVD simulation on the right

As with the initial test, early versions of the Ishihara imagery were sequenced in steps of three (off, on, on). One of the sets of the colours (red, darker green and darker brown) acts as off in the sequence, the other set (red-orange, lighter brown and lighter green) working as on. As the patterns and effects increased with difficulty, other sequences were developed and tested.

Once a section of the motion graphic was completed, the CVD simulation application was used to get a view of the effect planned for those with CVD (as shown in Figure 7). To be as inclusive as possible, the software was adjusted to simulate low to moderate CVD. When the test subjects were able to describe the effects as intentionally targeted to their visual acuities, the results were considered successful and that section of motion graphic deemed complete. For sections where the test subjects did not describe the intended effects, adjustments were made and the process repeated until the desired test subject response was attained. The motion graphics were then viewed using smart devices to ensure correct simulations were displayed. Those with typical colour vision viewed the motion graphics using the CVD simulation software; those with CVD used the recolouring software. The responses were positive in both situations.
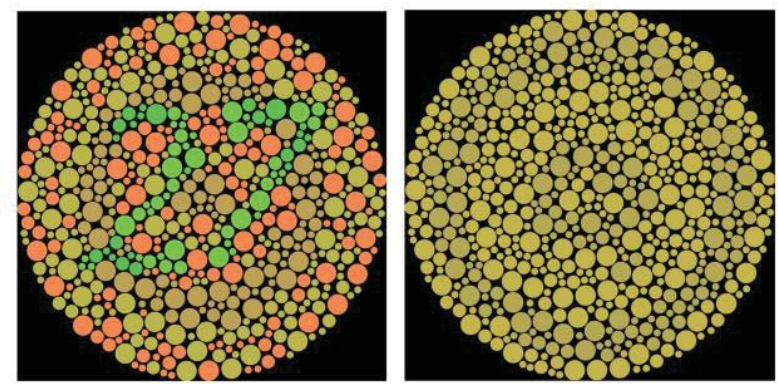

Figure 7: Still from Ishihara imagery motion graphic test Original colours on the left. CVD simulation on the right 


\section{CONCLUSION}

Applying an understanding of colour, artistic principles and computer science applications we were able to plan and execute motion graphics that had specific elements apparent only to targeted audiences of specific visual abilities. Using smart devices loaded with our simulation and recolouring applications, we shared those elements with larger audiences. Through these smart devices, those with typical colour vision were able to perceive aspects of the work specifically designed to be visible only to those with CVD. And, using the same smart devices, those with CVD were able to detect aspects of the artwork that would typically be hidden from them.

During this process, areas of refinement were identified. Test subjects were used that self identified as colour vision deficient. In the future, those CVD self-identified subjects will be screened to identify the type and severity of CVD to help ensure proper interpretation of responses.

Prototype art targeted those with typical colour vision and those with deuteranopia, the most common form of CVD. Through further development we believe that artwork can be designed that engages deuteranopia and protanopia simultaneously, with the expectation of creating mechanisms to include tritanopia (colloquially blue-yellow colour blindness).

It is our hope that through broad exhibition of the work, germane feedback can be amassed and the work thoughtfully progressed.

\section{REFERENCES}

Asada, K. (2011) The Day I Saw Van Gogh's Genius in a New Light. http://asada0.tumblr.com/ post/11517603099/the-day-i-saw-van-goghsgenius-in-a-new-light (17, March, 2014).

Australian Government. (2014) It's an Honour. http://www.itsanhonour.gov.au/honours/honour_roll /search.cfm?aus_award_id $=870096 \&$ search_type $=$ quick\&showlnd=true (17 March, 2014).

Brettel, H., Viénot, F., and Mollon, J.D. (1997) Computerized Simulation of Color Appearance for Dichromats. Journal of the Optical Society of America A, 14(10), 2647-2655.

Cole, B., and Harris, R. (2009) Colour Blindness Does Not Preclude Fame as an Artist: Celebrated Australian Artist Clifton Pugh was a Protanope. Clinical \& Experimental Optometry, 92, 421-428.

Flatla, D.R., and Gutwin, C. (2012) "So That's What You See!" Building Understanding With Personalized Simulations of Colour Vision Deficiency. In Proc. ASSETS '12, 167-174. ACM, New York, NY, USA.

Ishihara, S. (1917) Tests for Colour-Blindness. Pseudoisochromatic Plates.

Jefferson, L. and Harvey, R. (2006) Accommodating Color Blind Computer Users. In Proc. ASSETS '06, 40-47. ACM, New York, NY, USA.

Lyons, D. (2013) Testing Times. Printmaking Today, 22(4), 24-25.

Meyer, G.W. and Greenberg, D.P. (1988) ColorDefective Vision and Computer Graphics Displays. IEEE Computer Graphics \& Applications, 8, 28-40.

Sacks, O. (1995) An Anthropologist on Mars: Seven Paradoxical Tales. Alfred A. Knopf, USA.

Seewald Solutions. (2013) ColorBlindness SimulateCorrect. https://play.google.com/store/ apps/details?id=com. SeewaldSolutions. ColorBlindn essSimulator (21 March, 2014).

The Bullwinkle Show. (2011) Bullwinkle Show Intro. https://www.youtube.com/watch?v=ejgfnRvAOno (17, March, 2014).

The Gong Show. (2006) Oingo Boingo on the Gong Show.https://www.youtube.com/watch?v=vTRd1a5 MVMw (17, March, 2014). 